\title{
CNKSR1 gene defect can cause syndromic autosomal recessive intellectual disability
}

\author{
Somayeh Kazeminasab ${ }^{1}$ | Ibrahim Ihsan Taskiran² ${ }^{2}$ Zohreh Fattahi $^{1}$ | \\ Niloofar Bazazzadegan ${ }^{1}$ | Masoumeh Hosseini ${ }^{1}$ | Maryam Rahimi ${ }^{1}$ | Morteza Oladnabi ${ }^{1}$ | \\ Mohammad Haddadi $^{3}$ | Arzu Celik ${ }^{2}$ | Hans-Hilger Ropers ${ }^{4}$ | Hossein Najmabadi ${ }^{1}$ | \\ Kimia Kahrizi ${ }^{1}$
}

${ }^{1}$ Genetics Research Center, University of Social Welfare and Rehabilitation Sciences, Tehran, Iran

${ }^{2}$ Department of Molecular Biology and Genetics, Bogazici University, Istanbul, Turkey

${ }^{3}$ Department of Biology, Faculty of Science, University of Zabol, Zabol, Iran

${ }^{4}$ Department of Human Molecular Genetics, Max-Planck Institute for Molecular Genetics, Berlin, Germany

\section{Correspondence}

Kimia Kahrizi, Medical Genetics Research Center, University of Social Welfare and Rehabilitation Sciences, Daneshjoo Blvd, Koodakyar St., Evin, Tehran 1985713834, Iran. Email: ki.kahrizi@uswr.ac.ir;

kahrizi@yahoo.com

Funding information

Iranian National Science Foundation (INSF),

Grant/Award Number: 92035782
The advent of high-throughput sequencing technologies has led to an exponential increase in the identification of novel disease-causing genes in highly heterogeneous diseases. A novel frameshift mutation in CNKSR1 gene was detected by Next-Generation Sequencing (NGS) in an Iranian family with syndromic autosomal recessive intellectual disability (ARID). CNKSR1 encodes a connector enhancer of kinase suppressor of Ras 1, which acts as a scaffold component for receptor tyrosine kinase in mitogen-activated protein kinase (MAPK) cascades. CNKSR1 interacts with proteins which have already been shown to be associated with intellectual disability (ID) in the MAPK signaling pathway and promotes cell migration through RhoAmediated c-Jun N-terminal kinase (JNK) activation. Lack of CNKSR1 transcripts and protein was observed in lymphoblastoid cells derived from affected patients using qRT-PCR and western blot analysis, respectively. Furthermore, RNAi-mediated knockdown of cnk, the CNKSR1 orthologue in Drosophila melanogaster brain, led to defects in eye and mushroom body (MB) structures. In conclusion, our findings support the possible role of CNKSR1 in brain development which can lead to cognitive impairment.

\section{KEYWORDS}

autosomal recessive intellectual disability, CNKSR1, Drosophila, eye, mushroom bodies

\section{1 | INTRODUCTION}

In the last decade the field of medical genomics has been revolutionized by the advent of high-throughput sequencing technologies, and as a result, much attention has been paid to one of the greatest unsolved problems in health care, intellectual disability (ID). Intellectual disability has been defined as substantial limitations in intellectual functions, social and adaptive behaviors which begins before adulthood (Kaufman, Ayub, Vincent, 2010). Comprehensive epidemiological studies indicate the global prevalence of ID as $1 \%$ and $3 \%$ in developed and developing countries, respectively (Durkin, 2002; Ropers, 2008). Intellectual disability can be caused by genetic and environmental factors; however, genetic causes account for more than $50 \%$ of cases of ID (Kaufman et al., 2010). Early-onset cognitive impairment, or ID is divided into four categories including mild, moderate, severe and profound forms based on severity and affects about $85 \%, 10 \%, 4 \%$, and $2 \%$ of the world population, respectively (Maris, Barbato, Trott, \& Montano, 2013).

The autosomal recessive mode of inheritance accounts for approximately one-quarter of the total genetic cases of ID (Higgins, Pucilowska et al., 2004). Since 2007, to identify genetic defects in autosomal recessive intellectual disability (ARID), our group has initiated a systematic strategy in consanguineous Iranian families -with consanguinity rate $>40 \%$ - of homozygosity mapping, exon enrichment and nextgeneration sequencing. This ongoing project has revealed more than 50 variants in genes not previously identified to be linked to ARID (Motazacker et al., 2007; Garshasbi et al., 2008; Mir, 2009; Hu et al., 2011; Najmabadi et al., 2011; Pak, Garshasbi et al., 2011). 

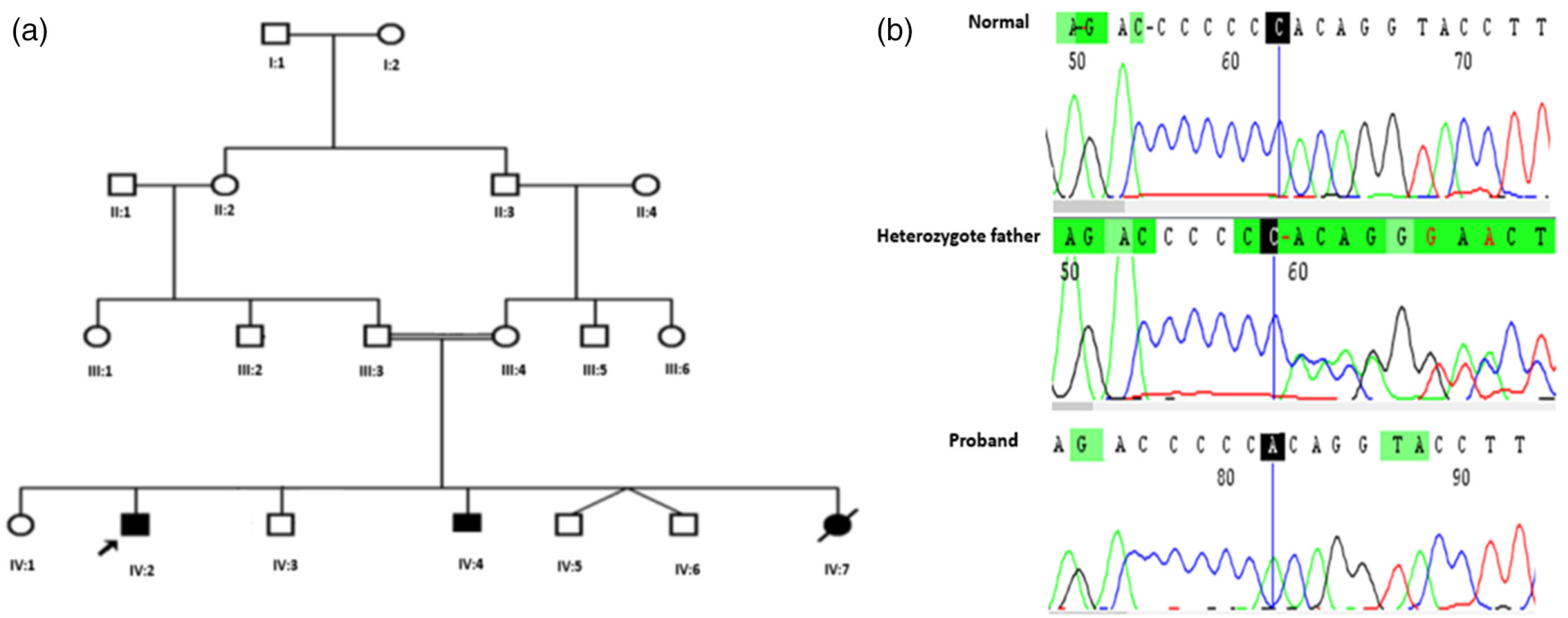

FIGURE 1 (a) Pedigree of four generations of the family investigated. Square symbols, male; round symbols, female. Filled symbols represent affected individuals. The arrow points to the proband in the family. (b) Representative sequence chromatograms for the CNKSR1 frameshift mutation, chr1:26382898-26382898del1; T282fs. A normal unrelated control was genotyped. The proband (IV:2) was homozygous for the mutation. The patient's father (III:3) was a heterozygous carrier of the T282fs mutation [Color figure can be viewed at wileyonlinelibrary.com]

Our group in the genetics research center (GRC) has reported several novel MRT loci (mental retardation loci) and genes for ARID which are the largest of its kind published to date. A novel frameshift mutation in CNKSR1 gene was detected in an Iranian family with syndromic ARID (Figure 1a). Genotyping data in this subject family (ID: 8500235) revealed five homozygous intervals located on chromosomes 1, 3, 4,13 , and 22. The largest and more prominent interval was located on chromosome 1 between SNP markers; rs10916983 and rs849961. Three candidate variants were identified by targeted exome sequencing in this interval of which only the variant in the CNKSR1 gene cosegregated with the disease(Figure 1b) (Najmabadi et al., 2011; Hu et al., 2018). The description of detected variants is given in Table 1.

The CNK protein family was first discovered to be a modifier of signaling pathways in Drosophila melanogaster. CNKSR1 is a ubiquitously expressed protein that is conserved from Drosophila to humans with a similar domain arrangement and is composed of several protein-protein interaction domains in signal transduction, SAM, CRIC, PDZ and PH (Therrien, Wong, Rubin, 1998; Claperon \& Therrien, 2007). CNKSR1 encodes a connector enhancer of kinase suppressor of Ras 1, which acts as a super scaffold for receptor tyrosine kinase in mitogen-activated protein kinase (MAPK) pathways (Jaffe, Aspenström et al., 2004).

The in vivo interaction of CNKSR1 with various proteins such as RhoA and RALGDS in the MAPK pathway, which have already been shown to be associated with ID suggested that CNKSR1 may also play a critical role in neuronal development (Newey, Velamoor, Govek, \& Van Aelst, 2005; Najmabadi et al., 2011).

CNKSR1 was introduced as a candidate gene in syndromic ID for the first time by Najmabadi et al., and there is no other reported association between the CNKSR1 gene and specific phenotypes or diseases to date. However, mutation in another member of the CNK protein family named CNKSR2, causes X-linked intellectual disability, seizure and other symptoms such as attention and language impairments. Moreover, a genome-wide association study, identified a genetic association between schizophrenia and the CNKSR2 gene (Schizophrenia Working Group of the Psychiatric Genomics, 2014; Vaags et al., 2014; Aypar, Wirrell, Hoppman, 2015).

Animal models are invaluable and powerful systems for identification of molecular mechanisms of genetically heterogeneous diseases such as ID (Scorza \& Cavalheiro, 2011). Among known ID genes, about $87 \%$ have a Drosophila orthologue and $76 \%$ show remarkable evolutionary conservation between humans and Drosophila (Inlow \& Restifo, 2004; Oortveld et al., 2013). In that respect, Drosophila has emerged as a powerful model organism which can be used to uncover the genetic basis, molecular pathways and treatment plans in early onset cognitive disorders (van der Voet, Nijhof et al., 2014).

The mushroom bodies (MBs) are a pair of prominent neuropil structures in the brain of Drosophila. They are comparable to the cerebral cortex and cerebellum-like structures in vertebrates and are essential for critical brain functions including learning, memory, attention and voluntary movements (Heisenberg, 1998; Farris, 2011).

In the present study, we attempted to elucidate the molecular effect of the CNKSR1 gene mutation in the cells and structures of MBs to further clarify its function and pathogenesis.

\section{2 | MATERIALS AND METHODS}

\section{1 | Ethics statement}

Ethical approval for this study was obtained from the Ethics Committee of the University of Social Welfare and Rehabilitation Sciences, Tehran, Iran. Informed consent was obtained from the living parents of patients.

\section{2 | Clinical ascertainment of family}

Clinical history-taking and physical examination were performed by a neurologist and the family was selected for molecular analysis based on the observed consanguinity. 
TABLE 1 Description of identified variants and bioinformatics pathogenicity prediction (Najmabadi, Hu et al., 2011)

\begin{tabular}{lllllll} 
Gene & Protein change & Logit score & SIFT & Polyphen2 & \multicolumn{2}{l}{$\begin{array}{l}\text { Iranian Controls } \\
\text { (800 Ind.) }\end{array}$} \\
\hline TRAPPC3 & R54Q & 2.53 & Damaging & Possibly damaging & NA & Status \\
MRPS15 & P193A & 2.53 & NA & Probably damaging & NA & Not co-segregating \\
\hline CNKSR1 & T282fs & 2.53 & NA & NA & NA & Co-segregating \\
BRCA2 & L1763V & 2.53 & Damaging & Benign & NA & Not co-segregating \\
\hline CRELD2 & Splice site & 2.53 & NA & NA & NA & Not co-segregating \\
\hline
\end{tabular}

\subsection{Establishment of lymphoblastoid cell lines and expression studies}

Obtaining a continuous source of cells and biomolecules is one of the major obstacles for genetic studies. To overcome this drawback, we established lymphoblastoid cell lines (LCLs) by in vitro infection of $B$ cells from peripheral blood with Epstein Barr Virus (EBV) from healthy and affected patients according to standard protocols. We isolated total cellular RNA from LCLs using the RNeasy Mini Kit (Qiagen, Hilden, Germany, cat. no. 74104). Complementary DNA (cDNA) was synthesized using a Revert Aid First Strand cDNA Synthesis Kit (Thermo Scientific \#K1622). UCSC Genome Browser on Human Feb. 2009 (GRCh37/hg19) Assembly indicates that human CNKSR1 has three protein coding isoforms. The presence of CNKSR1 transcript isoform 1 (NM: 006314) and transcript isoform 2 (NM: 001297647) was analyzed in LCLs. It should be noted that, because of complete similarity to other isoforms, transcript-specific primer design for isoform 3 was impossible. The SYBR Green-based qPCR in the ABI7500 instrument (Applied Biosystems, Foster City, CA) was performed to investigate the relative expression level of CNKSR1 transcripts in affected individuals versus controls. For accurate and reliable relative gene expression analysis, we normalized the results using TBP (TATA Binding Protein) specific primers. The data files were analyzed using 7500 Software v2.0.6.

\subsection{Protein extraction and Western blot analysis}

Based on a standard protocol, whole-cell proteins were extracted using RIPA (radio immunoprecipitation assay) lysis buffer. Proteins were electrophoresed on 10\% SDS-polyacrylamide gels and transferred to PVDF Western Blotting Membranes (Roche Applied Science, cat.no. 000000003010040001). We used two polyclonal antibodies,

TABLE 2 Primers used for gene expression analysis by qRT-PCR

\begin{tabular}{ll}
\hline Primers & Sequence $\left(5^{\prime}\right.$ to $\left.\mathbf{3}^{\prime}\right)$ \\
\hline CNKSR1isoform1 Fwd & GAAATTCACACCACCAGCAATTG \\
\hline CNKSR1isoform1 Rev & CATCCCACCACCACCTGC \\
\hline CNKSR1 isoform2 Fwd & GGCCTAGAATTCACACCACC \\
\hline CNKSR1isoform2 Rev & CCATCCCACCATGTCCCTC \\
\hline TBP Fwd & GTACCGCAGCTGCAAAATAT \\
\hline TBP Rev & TGGCTCTCTTATCCTCATGA \\
\hline cnk Fwd & TGCTCAACATACGGCCATACG \\
\hline cnk Rev & GCAGATTGTCGTTTTTCAGGTGA \\
\hline Rp49 Fwd & ATGCTAAGCTGTCGCACAAATG \\
\hline Rp49 Rev & GTTCGATCCGTAACCGATGT \\
\hline
\end{tabular}

Fwd, Forward; rev, Reverse. one against the C-terminus of CNKSR1 (NBP1-84420) and the other against the N-terminus of CNKSR1 (GTX31960). To control the variability, we normalized the immunoblot using a mouse monoclonal anti-beta Actin antibody (mAbcam 8226). The BM Chemiluminescence Western Blotting Kit (Roche Applied Science, cat. no. 11520709001) was used for detection.

\section{5 | Drosophila stocks and maintenance}

All of the Drosophila stocks were reared and maintained on standard wheat cream agar media supplemented with dried yeast granules under a light/dark cycle of $12: 12 \mathrm{~h}$ at $25 \pm 2{ }^{\circ} \mathrm{C}$. The elav ${ }^{\mathrm{c} 155}$-Gal4 (\#458) and UAS-RNAi ${ }^{\text {cnk }}$ (\#33366) were obtained from Bloomington Drosophila Stock Center. The OK107-Gal4 was a gift from Dr. BV Shyamala, Department of Zoology, University of Mysore, India. The

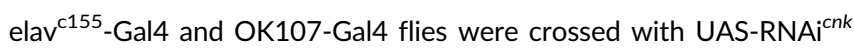
line in order to perform post-transcription cnk gene silencing in the brain and MBs, respectively. To achieve maximal GAL4/UAS expression, the fly stocks were kept at $29^{\circ} \mathrm{C}$ for $72 \mathrm{~h}$. The UAS-RNAi ${ }^{\text {cnk }}$ line served as control.

\subsection{Quantitative RT-PCR analysis of RNAi knockdown}

To evaluate the expression level of cnk transcript in Drosophila, virgin

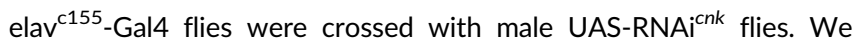
extracted total RNA from 50 homogenized heads of the progeny using TRIzol reagent. The relative expression level of cnk transcript was normalized by the endogenous expression level of $r p 49$. The primers were designed using the FlyPrimerBank online database and are shown in Table 2.

\subsection{Immunostaining and microscopy}

To determine the role of parental sex on phenotypic variation, we crossed flies in a reciprocal manner. We collected age and sex synchronized flies from OK107-Gal4 > UAS-RNAi ${ }^{\text {cnk }}$ and control. The adult brains were dissected, fixed in $4 \%$ paraformaldehyde, washed in PBS, 0.3\% Triton X-100 and then blocked in PAXD (PBS containing 5\%BSA, 0.3\% TritonX-100 and 0.3\% Sodium Deoxycholate) for 2 hours. The primary antibodies used were 1:200 mouse anti-FASII (1D4) and 1:20 rat-anti-Elav. The brains were incubated in primary antibodies overnight. Secondary antibodies conjugated with Alexa Fluor 488 and Alexa Fluor 647 were used at 1:400 dilution. Stained brains were mounted on slides in Vectashield mounting medium. These preparations were imaged using a Leica TCS SP8 confocal 

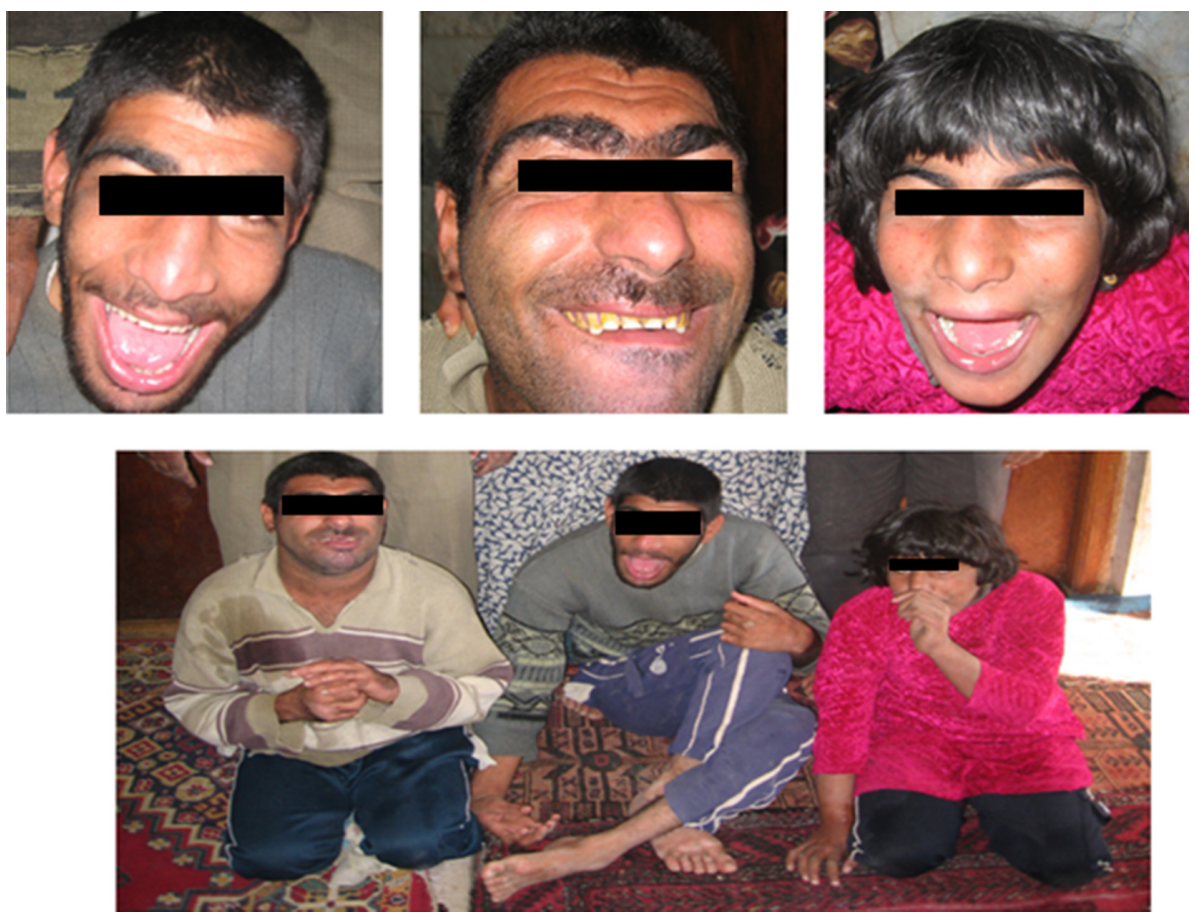

FIGURE 2 Clinical appearance of patients. Three patients in the subject's family with microcephaly, short stature, profound intellectual disability, myopia, strabismus, cerebellar hypoplasia, and quadrupedal gait [Color figure can be viewed at wileyonlinelibrary.com]

microscope. The images were analyzed and measured with Image software (version 1.51n). Finally, the Corrected Total Cell Fluorescence (CTCF) was calculated for each image using the following equation: $\mathrm{CTCF}=$ Integrated Density $-($ Area of ROI $) \times$ Mean Fluorescence of Background Readings.

\section{3 | RESULTS}

In Silico translation analysis for homozygous T282fs mutation in CNKSR1 gene was performed using the ExPASy Translate tool.
Analysis demonstrated that deletion of the $\mathrm{C}$ nucleotide at chr1:26382898 in exon 9 results in a frameshift that changes the reading frame of subsequent codons and also the introduction of premature termination codons (PTCs) downstream of the mutation which could lead to protein truncation or nonsense-mediated decay (NMD).

Comprehensive clinical assessment of the family being studied demonstrated microcephaly, short stature, profound intellectual disability, ataxia, aggression, and quadrupedal gait (Figure 2). Magnetic resonance imaging (MRI) revealed cerebellar hypoplasia in the patients. The clinical characteristics of all affected individuals are listed in Table 3.

TABLE 3 Clinical characteristics of affected individuals in the family

\begin{tabular}{llll} 
Feature & IV: 2 & IV: 4 & IV: 7 \\
\hline Gender & Male & Male & Female \\
\hline Age* $(\mathrm{Y})$ & 25 & 20 & 13 \\
\hline HC $(\mathrm{cm})$ & $49(-4.8 \mathrm{SD})$ & $51(-3.0 \mathrm{SD})$ & $47(-5.2 \mathrm{SD})$ \\
\hline Height $(\mathrm{cm})$ & $152(-3.4 \mathrm{SD})$ & $158(-2.6 \mathrm{SD})$ & $134(-3.3 \mathrm{SD})$ \\
\hline Head Control (M) & 10 & 8 & 7 \\
\hline Standing (M) & 30 & 30 & 24 \\
\hline Walking $(\mathrm{Y})$ & 6 & 5 & 6 \\
\hline Speaking $(\mathrm{Y})$ & 4 & 4 & 4 \\
\hline IQ & 15 & 19 & 16 \\
\hline MRI & Cerebellar hypoplasia & Cerebellar hypoplasia & Cerebellar hypoplasia \\
\hline Ataxia & + & + & + \\
\hline Gait & Quadrupedal gait & Quadrupedal gait & Quadrupedal gait \\
\hline Behavior & Aggression & Aggression & Aggression \\
\hline Eye & Strabismus/Myopia & Strabismus/Myopia & Strabismus/Myopia \\
\hline Speech & $<5$ words & $<10$ words & $<5$ words \\
\hline
\end{tabular}

*Age at the time of examination.

Y, years; HC, head circumference; SD, standard deviation; cm, centimeter; m, months; IQ, intelligence quotient; MRI, magnetic resonance imaging. 
(a)

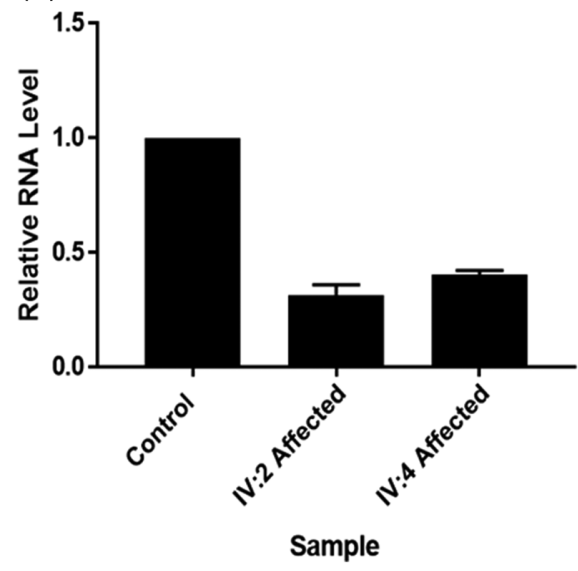

(b)

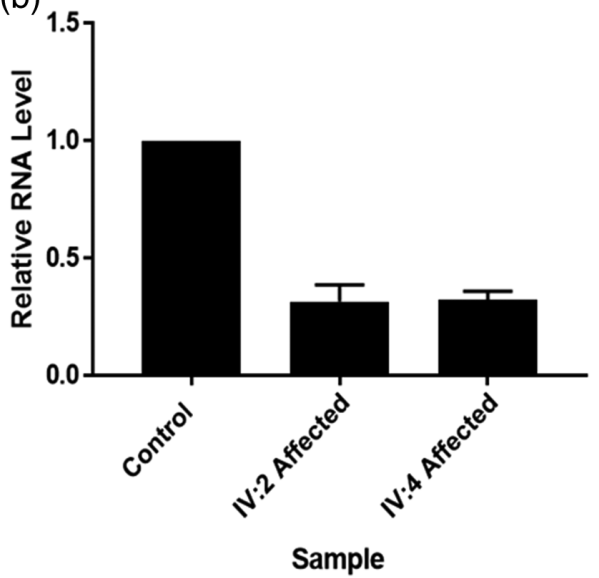

FIGURE 3 qRT-PCR analysis. qRT-PCR results using isoform-specific primers (a) NM: 006314, transcript isoform 1 and (b) NM: 001297647, transcript isoform 2, show a significant decrease in CNKSR1 transcripts in the affected individuals (IV:2, IV:4). Relative mRNA quantification was determined by comparison to reference gene expression levels in TBP (TATA Binding Protein)

To understand the pathophysiology of CNKSR1 mutation, we have investigated mRNA and CNKSR1 protein expression in cell lines derived from the patients and controls. Quantitative investigation of mRNA level expression by $\mathrm{qRT}-\mathrm{PCR}$ in derived cell lines indicated a reduction of CNKSR1 transcripts in the patient's cell line (Figure 3). There were no detectable and significant differences in expression level of CNKSR1 transcripts between the control and the heterozygous father. To survey CNKSR1 protein expression levels, we performed a western blot analysis on cell lysate from lymphoblastic cell lines derived from patients, heterozygous carrier and healthy unrelated control. We used two different antibodies, C-terminus (NBP1-84420) and N-terminus (GTX31960), which can bind to both isoform 1 and 2 simultaneously. First, we used antibodies against the $\mathrm{C}$-terminus. CNKSR1 $79 \mathrm{kDa}$ protein was detected in lysates from the controls and the heterozygous father (III:3) but not from the affected individuals (IV:2, IV:4). Therefore, we used CNKSR1 Nterminus antibody to further explore whether the protein is truncated or totally absent but we could not detect CNKSR1 in patients' cell lysates (IV:2, IV:4) (Figure 4). These observations indicated the lack of CNKSR1 protein in lymphoblastoid cells derived from affected patients with a homozygous T282fs mutation in the CNKSR1 gene which led to NMD.

For evaluation of RNA interference mediated cnk gene knockdown efficiency, we employed one of the most commonly used pan-neuronal drivers, elav ${ }^{\mathrm{c} 155}$-Gal4, which activates gene expression throughout the nervous system. Target mRNA reduction was corroborated by qRT-PCR (Figure 5a). In the Drosophila brain, mushroom bodies (MBs) were shown to be centers associated with learning, short- and long-term memory

(a)

Normal III:3

IV:2

IV:4

(a)

Father

Affected

Affected

B-actin

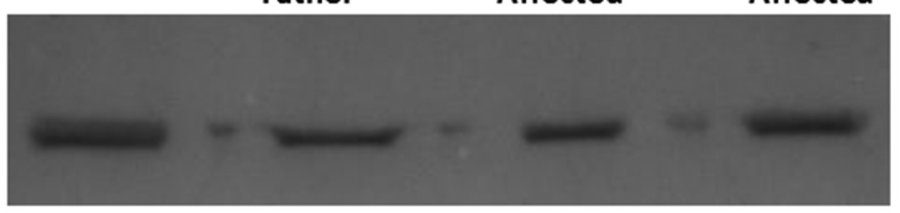

$55 \mathrm{kDa}$

$35 \mathrm{kDa}$

(b)

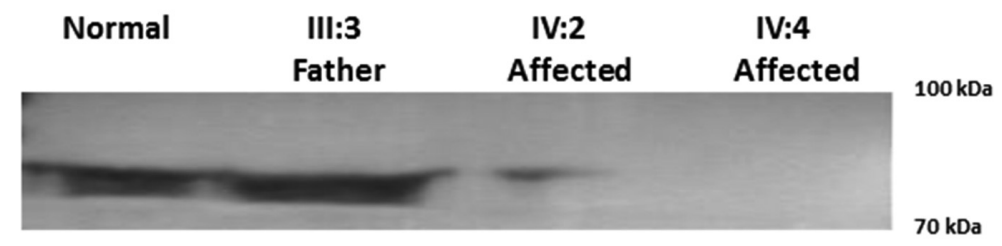

$100 \mathrm{kDa}$

CNKSR1

(c)

Normal

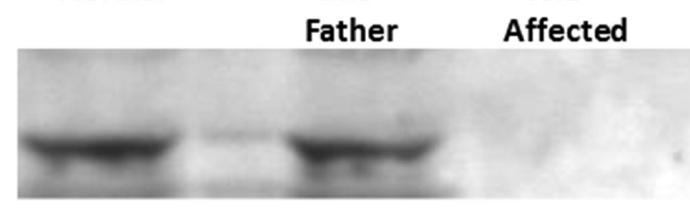

IV:4

Affected
$100 \mathrm{kDa}$

$70 \mathrm{kDa}$

FIGURE 4 Detection of $\beta$-actin and CNKSR1 protein levels by western blots of whole cell lysates from lymphoblastic cell lines. Results of western blotting using (a) mAbcam 8226 specific for $\beta$-actin, (b) NBP1-84420 antibody specific for CNKSR1 C-terminus, and (c) GTX31960 antibody specific for CNKSR1 $\mathrm{N}$-terminus are shown. $\beta$-actin shown as normalization control. The wild-type CNKSR1 is absent in the affected individuals (IV:2, IV:4) 


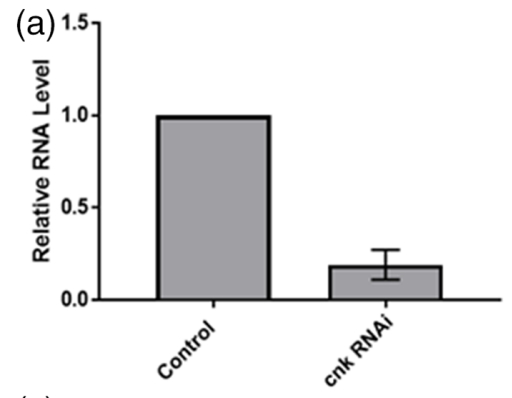

(c)

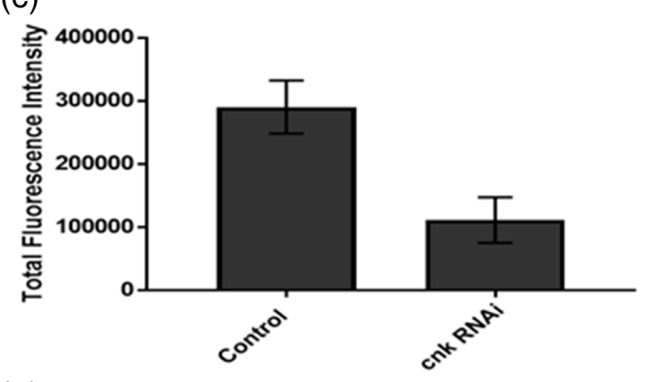

(d)

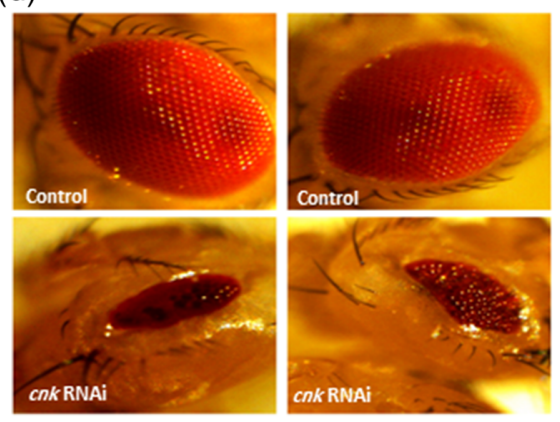

(b)

Control

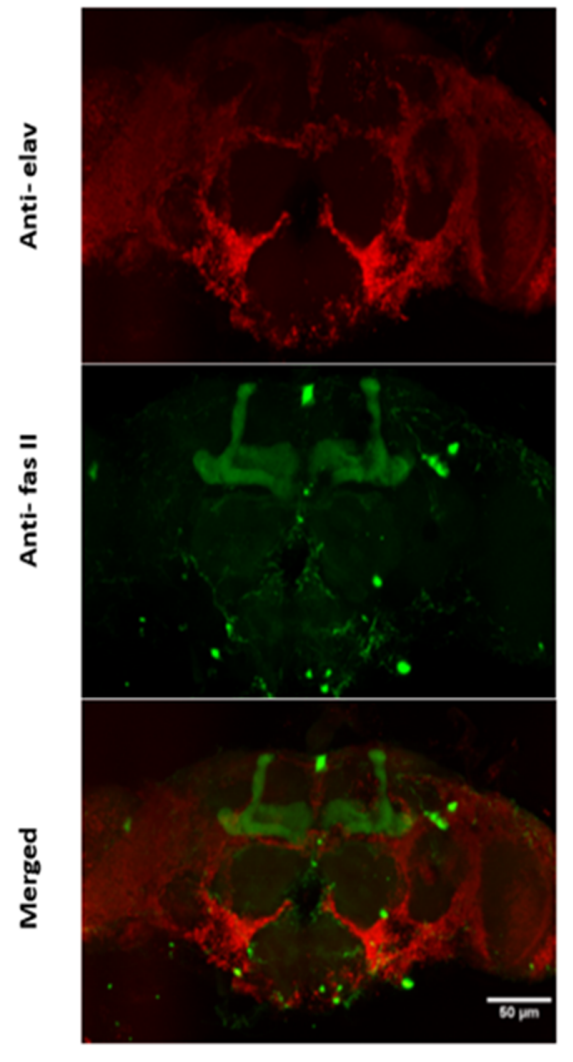

cnk RNAi

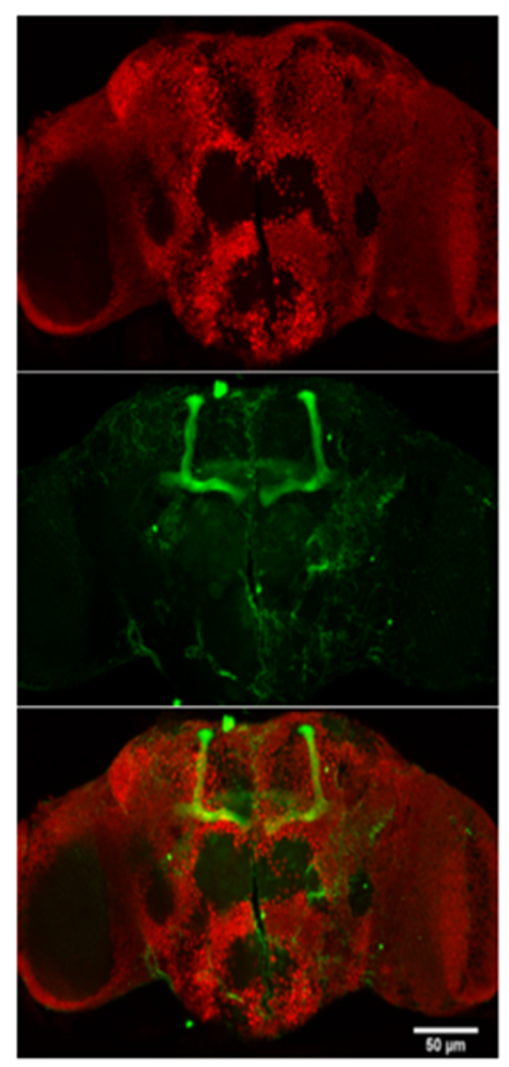

FIGURE 5 Analysis of $c n k$ downregulation in the Drosophila brain. (a) Confirmation of knockdown efficiency by qRT-PCR. Total RNA of fly head was extracted from Elavc155/+; UAS-RNAi ${ }^{\mathrm{cnk}} /+$. The results revealed that cnk transcript expression was only 20-25\% in knocked down flies. (b) Confocal microscopy of the brains and mushroom bodies from UAS-RNAicnk/+; OK107-Gal4/ + co-stained with Fasll and Elav. Cnk RNAi knocked down flies showed evidence of reduced size of mushroom bodies in comparison with UAS-RNAi ${ }^{\text {cnk }}$ control. (c) MBs from UAS-RNAi ${ }^{\text {cnk }}$ / +; OK107-Gal4/ + displayed a significant reduction in intensity of corresponding fluorescent signals. Scale bar, $50 \mu \mathrm{m}$. (d) Light microscopy eye structure of flies showed that RNAi-mediated knock down of cnk induced irregular and small eye phenotype in adults from UAS-RNAi ${ }^{\text {cnk } /+ \text {; }}$ OK107-Gal4/ + compared with UAS-RNAi ${ }^{\text {cnk }}$ control [Color figure can be viewed at wileyonlinelibrary.com]

(Heisenberg, 1998; Kurusu et al., 2000; Farris, 2011). Here, we investigated the possible effect of the cnk gene in MB architecture and organization using the UAS-Gal4 system with Fasll antibody to visualize neurons of the MB and elav to visualize all neuronal nuclei. Investigation of phenotypic characteristics of adult offspring of OK107-Gal4 and UAS$\mathrm{RNAi}^{\text {cnk }}$ crosses revealed a mutant eye phenotype which is greatly reduced in size, although not completely missing (Figure $5 \mathrm{~d}$ ). Afterwards, analysis of confocal images of the brains from six adult offspring demonstrated a significant reduction, approximately three-fold, in the size of MBs in cnk RNAi (Figure 5b, c). There were no significant differences between offspring from reciprocal crosses. This observation might be explained by the reduction of the number or degeneration of neurons due to the cnk knockdown in MBs, which may lead to cognitive impairments.

\section{4 | DISCUSSION}

CNKSR1 was first reported as a candidate gene in ARID in an Iranian family (Najmabadi et al., 2011). CNKSR1 gene encodes a scaffold protein for the MAPK signaling pathway, thereby playing an important role in assembling signaling molecules and enhancing the efficiency of intracellular signaling pathways. CNKSR1 contains four domains named SAM, CRIC, PDZ and PH which interact with various proteins in the signaling network (Therrien et al., 1998). The characteristics of CNKSR1 as one of the most important scaffold elements in maintaining the integrity of the MAPK pathway, cannot be ignored. The Rho family of GTPases in mammals comprises 20 members, and Cdc42, Rac1, and RhoA are the members which have been studied in detail. Rho GTPases accomplish several vital functions such as regulation of actin structures, cell adhesion and motility (Nobes \& Hall, 1995). The neuronal morphology of dendritic spines, which are commonly affected in ID, are modulated by Rho GTPases (Verpelli \& Sala, 2012; $\mathrm{Ba}$ et al., 2013). The PH domain of CNKSR1 interacts with Rho. Depletion of CNKSR1 or single amino acid substitutions in the $\mathrm{PH}$ domain impair Rho-dependent transcriptional activation (Jaffe, Aspenström et al., 2004). RalGDS encodes Ral guanine nucleotide dissociation stimulator which interacts with CNKSR1 by the N-terminal SAM and CRIC domains and acts in Ral signaling downstream of Ras 
(Therrien et al., 1999). RALDGS was identified as a candidate gene in ID (Najmabadi et al., 2011; Hill et al., 2016). On the other hand, results of previous studies indicate that alteration of gene expression in members of the MAPK pathway can relate to ID (Kaufman et al., 2010; McMillan et al., 2012). The reported relations with other proteins involved in the MAPK pathway could suggest CNKSR1 as a potential underlying factor in the etiology of ID. Thus, we believe that CNKSR1 is one of the critical members of the intracellular signaling pathways and its loss-of-function can cause cognitive perturbation. In silico translation analysis demonstrate that detected variant in CNKSR1 gene causes the introduction of a premature termination codon (PTC) in CNKSR1 mRNA which can lead to protein truncation or nonsense-mediated decay (NMD). However, according to the location of PTC, when the translation machinery halts at the first stop codon which is located far upstream of the last exon-exon junction, the NMD machinery, a sophisticated surveillance pathway in eukaryotic cell, can be engaged to initiate degradation of the mRNA to avoid the translation and accumulation of truncated proteins (Hsu et al., 2017). We observed significant reduction of CNKSR1 transcripts in patients compared to controls. The results of immunoblotting revealed the lack of CNKSR1 protein which were also in support of NMD.

Ephrins are transmembrane proteins containing a putative PDZ binding site at the $\mathrm{C}$-terminus which interacts with specific PDZ domain-containing proteins (Lin et al., 1999). Members of the ephrin family are involved in various developmental and homeostatic neural processes such as neurogenesis, synaptic plasticity, cell adhesion and migration (Klein, 2009). Ephrin B1 is a member of the ephrin family which interacts with CNKSR1 through the PDZ domain. This interaction promotes cell migration by inducing RhoA-mediated activation of the JNK signaling pathway (Cho et al., 2014). In vivo gain and loss of function of ephrin-B1 demonstrate that ephrin B1 plays a crucial role in migration of pyramidal neurons to the cerebral cortex $(\mathrm{Wu}$ et al., 2009; Dimidschstein et al., 2013). Furthermore, targeting a selected class of genes by RNAi reveals that PDZ domain-encoding genes such as cnk are involved in border cell migration in Drosophila (Aranjuez et al., 2012). Incorrect neuronal migration can cause several brain malformations and cognitive dysfunction (Gleeson \& Walsh, 2000). It is suggested that the reduction in number of neurons may affect neural circuit development of the brain, which is required for cognitive function and adaptive behavior. The MBs are a pair of neuropil structures in the brain of insects which consist of densely packed parallel neurons (Kenyon cells). MBs are structures similar to the cerebral cortex and cerebellum-like structures in vertebrates and are essential for important brain functions such as learning and memory, so that their size reduction in mutant flies have been reported to cause learning deficiencies (Heisenberg, 1998; Farris, 2011; Androschuk et al., 2015). According to this evidence, CNKSR1 and its orthologue in Drosophila play a critical role in cell migration process. So, we propose that any interruption in the expression of the CNKSR1 orthologue can lead to the reduction in MB size due to migration defects of the intrinsic neurons of the MBs and justifying the observed central nervous system phenotypes of intellectual impairment, significant microcephaly, cerebellar hypoplasia and quadrupedal gait in our patients.
The eye of Drosophila has been used productively as a model for clarification of the mechanisms of neural development and understanding signal transduction pathways (Domínguez, Wasserman et al., 1998; Voas \& Rebay, 2004). CNK scaffold protein is co-expressed and interacts with RAS in the Drosophila eye and overexpression of CNK or disruption of this interaction can lead to rough-eye phenotypes in Drosophila (Therrien et al., 1998; Therrien et al., 1999). The Ras-MAPK pathway has been shown to play an important role in cell proliferation and survival in the eye of Drosophila (Halfar et al., 2001). There are seven "master control" genes involved in the development of Drosophila's compound eyes - Pax6 homologs Eyeless (Ey) and Twin of Eyeless (Toy), the Pax2 homolog Eye Gone (Eyg), the Six homologs Sine Oculis (So) and Optix, Dachshund (Dac) and Eyes Absent (Eya) which are controlled by epidermal growth factor (EGF) signaling in the Ras-MAPK pathway (Kumar \& Moses, 2001; Lusk et al., 2017). Interestingly, three of these genes ey, toy, and dac, are expressed in the developing $\mathrm{MBs}$ and have critical functions in the structural formation of the MBs (Kurusu et al., 2000). We propose that MB-specific Gal4 driven knock-down of cnk can losen the integrity of the MAPK pathway which can also affect expression level of target genes such as ey, toy and dac _ eye master control genes in developing MBs. The observed irregular and dysmorphic eyes in Drosophila are likely caused by downregulation of $c n k$ in the eye due to leaky expression of the OK107-Gal4 driver, which is part of the eyeless enhancer. The results of ocular examination have revealed that the affected members of the family suffer from myopia and strabismus (Table 3).

In conclusion, the CNKSR1 mutation was introduced in an Iranian family with autosomal recessive intellectual disability. CNKSR1 encodes a conserved scaffold protein involved in MAPK signal transduction pathway. The absence of CNKSR1 abolishes its interactions with ID associated proteins and disrupts the integrity of the MAPK signaling pathway. Our primary hypothesis for the role of CNKSR1 in ID has been strengthened on the basis of the findings. We provide some evidence linking CNKSR1 dysfunction to neurological phenotypes in patients. We propose that clinical characteristics such as microcephaly, cerebellar hypoplasia in patients and the reduction in MBs size in Drosophila may be due to migration defects of the neuronal cells. In the following to confirm this hypothesis we need to improve our knowledge about the role of CNKSR1 in other animal models. Clearly, we have to challenge these finding using further experiments including CRISPR/Cas and RNAseq experiments in neural cell lines and suitable animal models to accumulate stronger evidence about the possible role of CNKSR1 in cognitive function.

\section{CONFLICT OF INTEREST}

The authors declare no conflict of interest.

\section{ACKNOWLEDGMENTS}

We would like to express our sincere gratitude to the family members who took part in this research and profoundly appreciate their collaboration which made this study possible. This article is part of Somayeh Kazeminasab's PhD project. This research was supported by Iranian National Science Foundation (INSF), grant no. 92035782. 


\section{REFERENCES}

Androschuk, A., Al-Jabri, B., \& Bolduc, F. V. (2015). From learning to memory: what flies can tell us about intellectual disability treatment. Frontiers in Psychiatry, 6, 68.

Aranjuez, G., Kudlaty, E., Longworth, M. S., \& McDonald, J. A. (2012). On the role of PDZ domain-encoding genes in Drosophila border cell migration. G3 (Bethesda, Md.)), 2(11), 1379-1391.

Aypar, U., Wirrell, E. C., \& Hoppman, N. L. (2015). CNKSR2 deletions: a novel cause of $\mathrm{X}$-linked intellectual disability and seizures. American Journal of Medical Genetics Part A, 167(7), 1668-1670.

Ba, W., van der Raadt, J., \& Kasri, N. N. (2013). Rho GTPase signaling at the synapse: Implications for intellectual disability. Experimental Cell Research, 319(15), 2368-2374.

Cho, H. J., Hwang, Y.-S., Mood, K., Ji, Y. J., Lim, J., Morrison, D. K., \& Daar, I. O. (2014). EphrinB1 interacts with CNK1 and promotes cell migration through c-Jun $\mathrm{N}$-terminal kinase (JNK) activation. The Journal of Biological Chemistry, 289(26), 18556-18568.

Claperon, A., \& Therrien, M. (2007). KSR and CNK: Two scaffolds regulating RAS-mediated RAF activation. Oncogene, 26(22), 3143-3158.

Dimidschstein, J., Passante, L., Dufour, A., Van Den Ameele, J., Tiberi, L., Hrechdakian, T., ... Jossin, Y. (2013). Ephrin-B1 controls the columnar distribution of cortical pyramidal neurons by restricting their tangential migration. Neuron, 79(6), 1123-1135.

Domínguez, M., Wasserman, J. D., \& Freeman, M. (1998). Multiple functions of the EGF receptor in Drosophila eye development. Current Biology $\mathrm{Cb}, 8(19), 1039-1048$.

Durkin, M. (2002). The epidemiology of developmental disabilities in lowincome countries. Mental Retardation and Developmental Disabilities Research Reviews, 8(3), 206-211.

Farris, S. M. (2011). Are mushroom bodies cerebellum-like structures? Arthropod Structure \& Development, 40(4), 368-379.

Garshasbi, M., Hadavi, V., Habibi, H., Kahrizi, K., Kariminejad, R., Behjati, F., ... Kuss, A. W. (2008). A defect in the TUSC3 gene is associated with autosomal recessive mental retardation. The American Journal of Human Genetics, 82(5), 1158-1164.

Gleeson, J. G., \& Walsh, C. A. (2000). Neuronal migration disorders: from genetic diseases to developmental mechanisms. Trends in Neurosciences, 23(8), 352-359.

Halfar, K., Rommel, C., Stocker, H., \& Hafen, E. (2001). Ras controls growth, survival and differentiation in the Drosophila eye by different thresholds of MAP kinase activity. Development, 128(9), 1687-1696.

Heisenberg, M. (1998). What do the mushroom bodies do for the insect brain? An introduction. Learning \& Memory (Cold Spring Harbor, N.Y.), 5(1-2), 1-10.

Higgins, J., Pucilowska, J., Lombardi, R., \& Rooney, J. (2004). Candidate genes for recessive non-syndromic mental retardation on chromosome 3p (MRT2A). Clinical Genetics, 65(6), 496-500.

Hill, W., Davies, G., Liewald, D., Payton, A., McNeil, C., Whalley, L., ... Pendleton, N. (2016). Examining non-syndromic autosomal recessive intellectual disability (NS-ARID) genes for an enriched association with intelligence differences. Intelligence, 54, 80-89.

Hu, H., Eggers, K., Chen, W., Garshasbi, M., Motazacker, M. M., Wrogemann, K., ... Bahman, I. (2011). ST3GAL3 mutations impair the development of higher cognitive functions. The American Journal of Human Genetics, 89(3), 407-414.

Hu, H., Kahrizi, Musante, K., Fattahi, L., Herwig, Z., Hosseini, R., Oppitz, M. C., ... Larti, F. (2018). Genetics of intellectual disability in consanguineous families. Molecular Psychiatry, 1.

Inlow, J. K., \& Restifo, L. L. (2004). Molecular and comparative genetics of mental retardation. Genetics, 166(2), 835-881.

Jaffe, A. B., Aspenström, P., \& Hall, A. (2004). Human CNK1 acts as a scaffold protein, linking Rho and Ras signal transduction pathways. Molecular and Cellular Biology, 24(4), 1736-1746.

Kaufman, L., Ayub, M., \& Vincent, J. B. (2010). The genetic basis of nonsyndromic intellectual disability: A review. Journal of Neurodevelopmental Disorders, 2(4), 182.

Klein, R. (2009). Bidirectional modulation of synaptic functions by Eph/ephrin signaling. Nature Neuroscience, 12(1), 15-20.
Kumar, J. P., \& Moses, K. (2001). Expression of evolutionarily conserved eye specification genes during Drosophila embryogenesis. Development Genes and Evolution, 211(8-9), 406-414.

Kurusu, M., Nagao, T., Walldorf, U., Flister, S., Gehring, W. J., \& FurukuboTokunaga, K. (2000). Genetic control of development of the mushroom bodies, the associative learning centers in the Drosophila brain, by the eyeless, twin of eyeless, and Dachshund genes. Proceedings of the National Academy of Sciences, 97(5), 2140-2144.

Lin, D., Gish, G. D., Songyang, Z., \& Pawson, T. (1999). The carboxyl terminus of $B$ class ephrins constitutes a PDZ domain binding motif. Journal of Biological Chemistry, 274(6), 3726-3733.

Lusk, J. B., Lam, V. Y., \& Tolwinski, N. S. (2017). Epidermal growth factor pathway signaling in Drosophila embryogenesis: Tools for understanding cancer. Cancers, 9(12), 16.

Maris, A. F., Barbato, I. T., Trott, A., \& Montano, M. A. E. (2013). Familial mental retardation: A review and practical classification. Ciência \& Saúde Coletiva, 18(6), 1717-1729.

McMillan, E. L., Kamps, A. L., Lake, S. S., Svendsen, C. N., \& Bhattacharyya, A. (2012). Gene expression changes in the MAPK pathway in both Fragile $X$ and Down syndrome human neural progenitor cells. American Journal of Stem Cells, 1(2), 154.

Mir, A., Kaufman, L., Noor, A., Motazacker, M. M., Jamil, T., Azam, M., ... Nasr, T. (2009). Identification of mutations in TRAPPC9, which encodes the NIK-and IKK- $\beta$-binding protein, in nonsyndromic autosomal-recessive mental retardation. The American Journal of Human Genetics, 85(6), 909-915.

Motazacker, M. M., Rost, B. R., Hucho, T., Garshasbi, M., Kahrizi, K., Ullmann, R., ... Goswami, C. (2007). A defect in the ionotropic glutamate receptor 6 gene (GRIK2) is associated with autosomal recessive mental retardation. The American Journal of Human Genetics, 81(4), 792-798.

Najmabadi, H., Hu, H., Garshasbi, M., Zemojtel, T., Abedini, S. S., Chen, W., ... Jamali, P. (2011). Deep sequencing reveals 50 novel genes for recessive cognitive disorders. Nature, 478(7367), 57-63.

Newey, S. E., Velamoor, V., Govek, E. E., \& Van Aelst, L. (2005). Rho GTPases, dendritic structure, and mental retardation. Journal of Neurobiology, 64(1), 58-74.

Nobes, C. and A. Hall (1995). Rho, rac and cdc42 GTPases: Regulators of actin structures, cell adhesion and motility, Portland Press Limited.

Oortveld, M. A., Keerthikumar, S., Oti, M., Nijhof, B., Fernandes, A. C., Kochinke, K., ... Eshuis, L. (2013). Human intellectual disability genes form conserved functional modules in Drosophila. PLoS Genetics, 9(10), e1003911.

Pak, C., Garshasbi, M., Kahrizi, K., Gross, C., Apponi, L. H., Noto, J. J., ... Behjati, F. (2011). Mutation of the conserved polyadenosine RNA binding protein, $\mathrm{ZC} 3 \mathrm{H} 14 / \mathrm{dNab} 2$, impairs neural function in Drosophila and humans. Proceedings of the National Academy of Sciences, 108(30), 12390-12395.

Ropers, H. H. (2008). Genetics of intellectual disability. Current Opinion in Genetics \& Development, 18(3), 241-250.

Schizophrenia Working Group of the Psychiatric Genomics, C. (2014). Biological insights from 108 schizophrenia-associated genetic loci. Nature, 511(7510), 421-427.

Scorza, C. A., \& Cavalheiro, E. A. (2011). Animal models of intellectual disability: towards a translational approach. Clinics (Sao Paulo), 66 (Suppl 1), 55-63.

Therrien, M., Wong, A. M., Kwan, E., \& Rubin, G. M. (1999). Functional analysis of CNK in RAS signaling. Proceedings of the National Academy of Sciences of the United States of America, 96(23), 13259-13263.

Therrien, M., Wong, A. M., \& Rubin, G. M. (1998). CNK, a RAFbinding multidomain protein required for RAS signaling. Cell, 95(3), 343-353.

Vaags, A. K., Bowdin, S., Smith, M. L., Gilbert-Dussardier, B., BrockeHolmefjord, K. S., Sinopoli, K., ... Minassian, B. A. (2014). Absent CNKSR2 causes seizures and intellectual, attention, and language deficits. Annals of Neurology, 76(5), 758-764.

van der Voet, M., Nijhof, B., Oortveld, M. A., \& Schenck, A. (2014). Drosophila models of early onset cognitive disorders and their clinical applications. Neuroscience \& Biobehavioral Reviews, 46, 326-342. 
Verpelli, C., \& Sala, C. (2012). Molecular and synaptic defects in intellectual disability syndromes. Current Opinion in Neurobiology, 22(3), 530-536.

Voas, M. G., \& Rebay, I. (2004). Signal integration during development: insights from the Drosophila eye. Developmental Dynamics, 229(1), 162-175.

Wu, C., Qiu, R., Wang, J., Zhang, H., Murai, K., \& Lu, Q. (2009). ZHX2 Interacts with Ephrin-B and regulates neural progenitor maintenance in the developing cerebral cortex. Journal of Neuroscience, 29(23), 7404-7412.
How to cite this article: Kazeminasab S, Taskiran II, Fattahi Z, et al. CNKSR1 gene defect can cause syndromic autosomal recessive intellectual disability. Am J Med Genet Part B. 2018;

177B:691-699. https://doi.org/10.1002/ajmg.b.32648 